\title{
Laser capture microdissection in pathology
}

\author{
Falko Fend, Mark Raffeld
}

\begin{abstract}
The molecular examination of pathologically altered cells and tissues at the DNA, RNA, and protein level has revolutionised research and diagnostics in pathology. However, the inherent heterogeneity of

of the technique of LCM, summarises current applications and new methodical approaches, and tries to give a perspective on future developments. In addition, LCM is compared with other recently developed laser microdissection techniques.
\end{abstract} primary tissues with an admixture of various reactive cell populations can affect the outcome and interpretation of molecular studies. Recently, microdissection of tissue sections and cytological preparations has been used increasingly for the isolation of homogeneous, morphologically identified cell populations, thus overcoming the obstacle of tissue complexity. In conjunction with sensitive analytical techniques, such as the polymerase chain reaction, microdissection allows precise in vivo examination of cell populations, such as carcinoma in situ or the malignant cells of Hodgkin's disease, which are otherwise inaccessible for conventional molecular studies. However, most microdissection techniques are very time consuming and require a high degree of manual dexterity, which limits their practical use. Laser capture microdissection (LCM), a novel technique developed at the National Cancer Institute, is an important advance in terms of speed, ease of use, and versatility of microdissection. LCM is based on the adherence of visually selected cells to a thermoplastic membrane, which overlies the dehydrated tissue section and is focally melted by triggering of a low energy infrared laser pulse. The melted membrane forms a composite with the selected tissue area, which can be removed by simple lifting of the membrane. LCM can be applied to a wide range of cell and tissue preparations including paraffin wax embedded material. The use of immunohistochemical stains allows the selection of cells according to phenotypic and functional characteristics. Depending on the starting material, DNA, good quality mRNA, and proteins can be extracted successfully from captured tissue fragments, down to the single cell level. In combination with techniques like expression library construction, cDNA array hybridisation and differential display, LCM will allow the establishment of "genetic fingerprints" of specific pathological lesions, especially malignant neoplasms. In addition to the identification of new diagnostic and prognostic markers, this approach could help in establishing individualised treatments tailored to the molecular profile of a tumour. This review provides an overview (F Clin Pathol 2000;53:666-672)

Keywords: laser capture microdissection; RNA analysis; DNA analysis; gene expression; profiling; immunohistochemistry

The molecular analysis of DNA, RNA, and protein derived from diagnostic tissue specimens has revolutionised pathology and led to the identification of a broad range of diagnostic and prognostic markers influencing our day to day decision making. Techniques such as Southern blot analysis and especially the polymerase chain reaction (PCR) are an integral part of the diagnostic repertoire of pathology. However, the reliability of tests based on tissue or cell extracts often depends crucially on the relative abundance of the cell population in question, and sampling errors or a large number of "contaminating" cells as a result of the inherent complexity of primary tissues can lead to false negative results. This is especially true for the examination of early or precursor lesions such as carcinoma in situ and epithelial dysplasias, which are of special interest, because they could shed light on the first, seminal steps of carcinogenesis. Whereas some genetic alterations, such as tumour specific chromosomal translocations, can be detected by PCR even if they are present only in a small minority of examined cells others, such as clonal immunoglobulin or $\mathrm{T}$ cell receptor gene rearrangements or loss of heterozygosity at tumour suppressor gene loci, require that the proportion of altered cells exceeds a certain threshold. In situ hybridisation is a valuable alternative to in vitro studies for the analysis of certain alterations, such as numerical and major structural chromosomal aberrations, viral infection, or aberrant gene expression, but in situ techniques are not universally applicable, and are time consuming and sometimes technically difficult. Furthermore, their relatively low sensitivity precludes the examination of single copy genes or low abundance transcripts. In situ PCR might help to improve the sensitivity for certain targets, but does not allow quantification and still poses significant technical problems - for example, in terms of specificity. Cell cultures and cell lines provide pure populations, which can be studied under controlled conditions. However, because of the different microenvironment-with the lack of contact and interaction with stromal elements-gene expression patterns of cultured cells can be very different to primary 
tissues. Furthermore, most permanent tumour cell lines are derived from late stage cancers already showing a far advanced genotype with multiple aberrations that might not be representative of earlier clinical stages.

In combination with sensitive analytical procedures such as PCR, microdissection of histological sections or cytological preparations has recently gained importance as a tool to obtain purified cell populations from complex primary tissues. From relatively crude, manual microdissection to micromanipulator based approaches, a wide range of techniques has been described and has been useful in medical research-for example, the identification of clonal genetic abnormalities in preneoplastic lesions or the unveiling of the cell lineage and clonality of Hodgkin's cells. ${ }^{1-10}$ More recently, the precision of lasers has been exploited for microdissection. ${ }^{11-17}$ One of these techniques is laser microbeam microdissection (LMM), which uses a pulsed UV laser with a small beam focus to cut out areas or cells of interest by photoablation of adjacent tissue. ${ }^{11}{ }^{13} 15$ The second technique is laser capture microdissection (LCM), which was developed at the National Cancer Institute of the National Institutes of Health, Bethesda, and has rapidly found widespread interest as an attractive addition to the repertoire of microdissection techniques. ${ }^{16}{ }^{18-21}$ The development of LCM was propelled by its integration into the "cancer genome anatomy project" (CGAP) sponsored by the National Cancer Institute, which intends to compile a catalogue of genes expressed by normal, preneoplastic, and neoplastic human tissues. ${ }^{22}$ To develop high quality expression libraries from human cancers and their precursor lesions, microdissection was seen as an important tool to provide pure cell samples. LCM fulfills the requirements in speed, precision, and versatility necessary for such an ambitious project. The first description of LCM was soon followed by the development of a commercially available LCM microscope. ${ }^{18}$ Currently, the second generation of this instrument (PixCell II; Arcturus Engineering, Mountain View, California, USA) is on the market.

\section{Principles and technical basis of LCM}

LCM is based on the selective adherence of visually targeted cells and tissue fragments to a thermoplastic membrane activated by a low energy infrared laser pulse (fig 1). The system consists of an inverted microscope, a solid state near infrared laser diode, a laser control unit, a joy stick controlled microscope stage with a vacuum chuck for slide immobilisation, a CCD camera, and a colour monitor. The LCM microscope is usually connected to a personal computer for additional laser control and image archiving. The thermoplastic membrane used for the transfer of selected cells has a diameter of approximately $6 \mathrm{~mm}$ and is mounted on an optically clear cap, which fits on standard $0.5 \mathrm{ml}$ microcentrifuge tubes for further tissue processing.

The cap is suspended on a mechanical transport arm and placed on the desired area of the dehydrated tissue section under standard
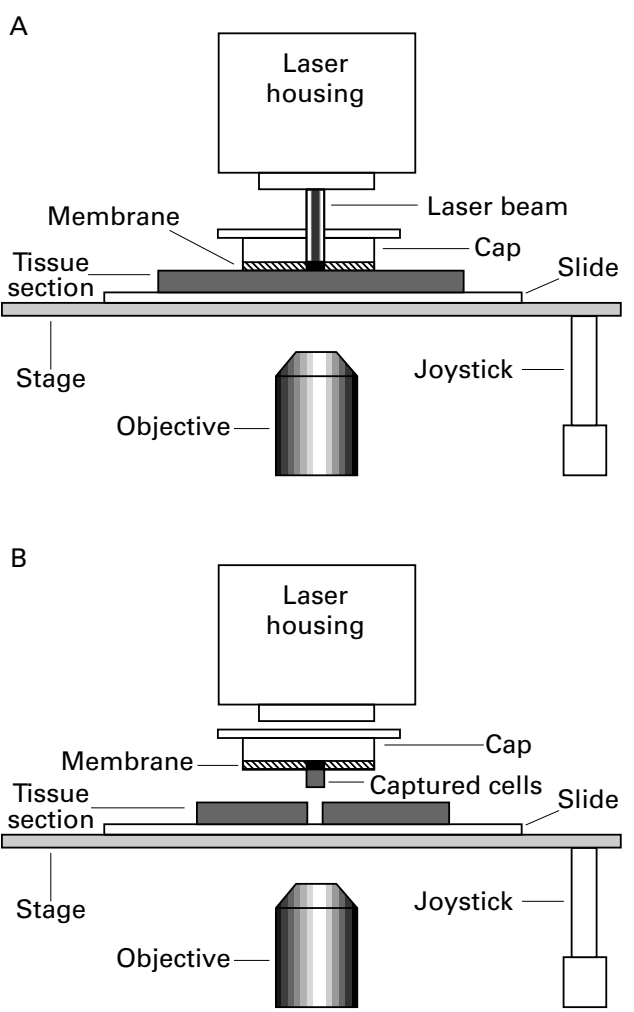

Figure 1 Schematic representation of laser capture microdissection. (A) Activation of the laser leads to focal melting of the polymer membrane. (B) Lifting of the cap selectively detaches the cells adherent to the activated membrane.

pressure. After visual selection of the desired cells guided by a positioning beam, laser activation leads to focal melting of the ethylene vinyl acetate (EVA) membrane, which has its absorption maximum near the wavelength of the laser. The melted polymer expands into the section and fills the extremely small hollow spaces present in the tissue. The polymer resolidifies within milliseconds and forms a composite with the tissue. The adherence of the tissue to the activated membrane exceeds the adhesion to the glass slide and allows selective removal of the desired cells. Laser impulses usually between 0.5 and $5 \mathrm{~ms}$ in duration can be repeated multiple times across the whole cap surface, which allows the rapid isolation of large numbers of cells. The selected tissue fragments are harvested by simple lifting of the cap, which is then transferred to a microcentrifuge tube containing the buffer solutions required for the isolation of the molecules of interest.

The minimum diameter of the laser beam of the LCM microscope is currently $7.5 \mu \mathrm{m}$, the maximum diameter $30 \mu \mathrm{m}$. Under standard working conditions, the area of polymer melting corresponds quite exactly to the laser spot size. Because most of the energy is absorbed by the membrane, the maximum temperatures reached by the tissue upon laser activation are in the range of $90^{\circ} \mathrm{C}$ for several milliseconds, thus leaving biological macromolecules of interest intact. ${ }^{23}$ The low energy of the infrared laser also avoids potentially damaging photochemical effects. 


\section{Advantages and disadvantages of LCM compared with other laser \\ microdissection techniques}

The most important advantage of LCM is its speed, combined with its precision and versatility. Depending on the chosen laser spot size, the architectural features of the tissue and the desired precision of the microdissection, thousands of cells can be collected within a few minutes. LCM is in orders of magnitude faster than micromanipulator based microdissection techniques and does not require manual dexterity because it is a "no touch" technique. The morphology of both the captured cells as well as the residual tissue is well preserved, allowing control and documentation of tissue representativity at all stages of the procedure. The isolated cells are attached firmly to the cap, minimising the danger of tissue loss. In contrast, most other microdissection techniques, with the exception of LMM with laser pressure catapulting, ${ }^{15}$ require the removal of the isolated cells with the help of a needle tip or a microcapillary - a precarious step requiring skill and practice. Because LCM is very fast and does not destroy adjacent tissues, several tissue components can be sampled sequentially from the same slide, such as normal and neoplastic cells. Owing to the easy handling of the LCM microscope and the virtual absence of time consuming calibration and adjustment, the learning curve is short and LCM can be integrated easily into procedures for molecular genetic tissue diagnostics.

LCM can be applied to a wide range of cell and tissue preparations. Even stained, archival sections can be microdissected successfully after removal of the coverslip.

The few limitations of LCM mostly reflect the difficulties of microdissection in general. Although sufficient for most purposes, the optical resolution of routinely stained, dehydrated tissue sections without coverslip is limited, and may make precise dissection of cells from certain complex tissues with lack of architectural features, such as lymphoid tissues or diffusely infiltrating carcinomas, almost impossible. This problem can be circumvented by special stains, in particular immunohistochemistry, which help to highlight the cell population to be isolated or avoided. ${ }^{24}{ }^{25}$

The minimum laser spot size of $7.5 \mu \mathrm{m}$ poses a limit to the precision of single cell or subcellular microdissection. Small cells may be difficult to isolate without contaminating fragments of adjacent cells. In addition, as a result of the large tissue contact area of the cap, the risk of contamination through rare, non-specifically adherent cells may be more important when LCM is performed at the single cell level. Although LCM was not primarily designed for single cell analysis, several studies showed the feasibility of analysing single or few cells procured by LCM from both tissue sections and cytological preparations. ${ }^{26-29}$ The latter allow faster and more precise collection of pure cell populations by LCM because the cells are already physically separated. Maitra et al developed a technique for the separation and isolation of epithelial cell aggregates (EASI) by spreading out scrapings of fresh tissues on glass slides. This procedure separates epithelial cell complexes from accompanying stroma and allows the rapid and precise procurement of cells by LCM without the necessity for tissue sectioning. ${ }^{28}$

A problem occasionally encountered in LCM is failure to remove the selected cells from the slide. This can result from a lack of adherence of the cells to the membrane, usually because of incomplete tissue dehydration or a laser setting that is too low for complete permeation of the melted polymer into the section. On the other hand, increased adherence of the section to the slide can prevent the removal of the targeted cells. This is mainly encountered in frozen sections, if they are subjected to prolonged drying, whereas paraffin wax sections normally do not require special handling. Several groups have reported detailed protocols to ensure optimal transfer conditions for a broad range of specimens, including immunohistochemically stained ones. ${ }^{16} 2426-28$

The other commercially available, advanced laser microdissection technology is laser microbeam microdissection (PALM; Mikrolaser Technologie, Bernried, Germany). In contrast to LCM, the tissue is cut out from the surrounding structures by a highly focused UV laser beam with the help of a motorised stage. The tissue is either removed by a needle tip or by laser pressure catapulting, which allows contact free collection of tissue fragments in the cap of a microcentrifuge tube. ${ }^{15}$ For many applications, tissue sections are mounted on a thin supporting membrane, which is also cut by the laser beam, and allows the recovery of larger, intact fragments of tissue with retained morphology. ${ }^{13}$

Both LCM and LMM with laser pressure catapulting are important technical advances in microdissection, and future technical developments will improve the performance of both systems. Which of these two systems is more suited for a certain application depends largely on the requirements for tissue collection. High speed, easy handling, and good control and documentation of dissected tissue make LCM an ideal tool for the rapid collection of larger amounts of tissue and the pooling of larger numbers of single cells. LMM can be more time consuming and requires greater skills, but the smaller laser beam diameter, the motorised stage, and the absence of a large surface area with tissue contact (like the LCM cap) is probably better suited for very precise microdissection at the single cell and subcellular level.

\section{Applications of laser microdissection}

Although the first descriptions of the use of laser light for microdissection of tissue were published in $1976,{ }^{30}{ }^{31}$ it was not until the introduction of efficient analytical methods for small amounts of biological material that these techniques found widespread use.

DNA ANALYSIS

Microdissection is an established method to obtain purified cell populations for the analysis 
of genetic alterations at the DNA level from both fresh and fixed tissues and cell preparations. Many different microdissection techniques have been used for the detection of loss of heterozygosity (LOH) in invasive neoplasms, epithelial precursor lesions in various organs including breast, oesophagus, and prostate, and in normal tissue adjacent to neoplastic lesions. ${ }^{12632-34}$ In combination with whole genome amplification, ${ }^{17}{ }^{35}$ comparative genomic hybridisation has been applied successfully to microdissected neoplastic and preneoplastic lesions of breast, cervix, and oral epithelium. ${ }^{36-38}$

LOH studies and other DNA based analyses can be performed with ease on cell and tissue fragments captured by LCM. ${ }^{16}$ Captured cells are detached from the membrane by proteinase digestion, and standard single step PCR protocols can be applied if enough cells have been collected. Because of the small amounts of tissue used, time consuming purification steps such as phenol/chloroform extraction are usually unnecessary.

The analysis of microdissected endocrine tumours played an instrumental role in identifying the multiple endocrine neoplasia type 1 (MEN1) gene by positional cloning. ${ }^{39}$ With newly identified polymorphic markers, haplotyping of affected families and $\mathrm{LOH}$ analysis of 200 microdissected endocrine tumours narrowed down the interval to $300 \mathrm{~kb}$, and further analysis of transcripts located in this region in MEN1 kindreds allowed the identification of this new tumour suppressor gene. LCM has also been used to identify LOH in ovarian neoplasms. The authors found allelic loss at chromosome $8 \mathrm{p} 21$ in a higher proportion $(50 \%)$ of cases than previous studies, probably because of the homogeneous cell populations obtained by microdissection. ${ }^{40}$ Darling et al used LCM to analyse the partial re-expression of type XVII collagen in a patient with generalised atrophic benign epidermolysis bullosa, who was germ line homozygous for a $2 \mathrm{bp}$ deletion in the COL17A1 gene. They showed that microdissected keratinocytes overlying areas with type XVII collagen re-expression had an additional frame restoring mutation, correcting nonsense mediated mRNA decay and therefore creating mosaicism for this gene product. ${ }^{41}$ Recently, LCM has also been used to demonstrate intratumoral hetrogeneity of p53 mutations in aflatoxin induced murine lung tumours. $^{42}$

Fend et al analysed cases of malignant non-Hodgkin's lymphoma with two phenotypically and morphologically distinct cell populations in the same tumour site. Sequencing of rearranged immunoglobulin genes amplified from the two different populations obtained by LCM of immunostained slides demonstrated the presence of two unrelated clones in all cases, although PCR analysis of DNA obtained from whole sections had not detected biclonality in any of the tumours. ${ }^{25} \mathrm{LCM}$ has also been used successfully to microdissect ReedSternberg (RS) like cells from peripheral T cell lymphomas. Amplification of immunoglobulin heavy chain genes from pooled groups of microdissected RS like cells, which expressed CD20 and Epstein-Barr virus (EBV) antigens, showed patterns suggestive of a polyclonal or oligoclonal EBV transformed B cell population, unrelated to the neoplastic $\mathrm{T}$ cell clone. ${ }^{29}$ The two latter studies demonstrate that routinely immunostained paraffin wax embedded sections mounted on charged slides can be used for LCM without interfering with the transfer of captured cells or subsequent PCR analysis.

Because it can rapidly sample large numbers of purified cells from heterogeneous tissues, LCM is also a promising tool for other DNA based analyses, such as comparative genomic hybridisation.

\section{GENE EXPRESSION ANALYSIS}

The analysis of tissue or cell specific gene expression is important in many fields of biological and biomedical research. The identification of gene expression patterns related to neoplastic transformation, inflammation, or tissue repair could have far reaching consequences not only for diagnostic and prognostic purposes, but also for preventive medicine by identifying subclinical stages of disease, and for novel treatments tailored to specific genetic alterations. ${ }^{43}$

However, tissue heterogeneity can make it very difficult to assign expressed genes to specific cell populations if gross tissue extracts are used as an mRNA source. Confirmation by in situ techniques, such as mRNA in situ hybridisation or immunohistochemistry, might not always be possible and is laborious and time consuming when large numbers of messages need to be examined. Furthermore, mRNA in situ hybridisation lacks sensitivity for the detection of low abundance transcripts. Therefore, many groups have tried to develop microdissection protocols that yield mRNA of sufficient quality for the subsequent analysis of gene expression. In contrast to DNA, mRNA is more sensitive to fixation, is quickly degraded by ubiquitous RNases, and requires stringent RNase free conditions during specimen handling and preparation. Despite these limitations, several groups have recovered good quality mRNA from microdissected samples by reverse transcription PCR (RT-PCR), down to the single cell level. ${ }^{3121444}$ Although most authors used fresh tissue, successful mRNA amplification by nested RT-PCR has also been reported for single cells isolated from paraffin wax embedded archival tissue by LMM and for methacarn fixed, paraffin wax embedded tissue by LCM. ${ }^{15}{ }^{45}$ Nevertheless, frozen tissue yields higher quality mRNA and should be used whenever possible to ensure optimal recovery of low abundance messages.

LCM offers several advantages for mRNA analysis. Its superior speed allows sampling of large numbers of cells without appreciable RNA degradation. This should help to reduce artifacts caused by a high number of amplification cycles or a lack of reproducibility as a result of variations of gene expression in small cell samples. In addition, the dehydration of the tissue section during LCM probably inhibits 
the activity of tissue RNases, in contrast to some techniques where microdissection is performed on buffer covered sections. Several groups have tried to assess the optimal conditions for RNA recovery from tissue subjected to LCM. ${ }^{16242846}$ Although the reported protocols differ in some aspects, the type of fixation, time in aqueous media, RNase free conditions during staining and tissue preparation, and probably also the tissue type are relevant factors. In general, precipitating fixatives such as acetone or ethanol give better results than crosslinking agents such as formaldehyde. Conventional stains like haematoxylin/ eosin can be performed in a very short time and do not greatly influence RNA recovery. For many applications, these stains are sufficient for the morphological identification and isolation of distinct cell populations for mRNA analysis. ${ }^{47-49}$ To perform precise LCM of tissues showing a lack of architectural features identifiable by morphology alone, immunohistochemical staining protocols designed to minimise RNA loss during the procedure have been developed. Immunohistochemistry of frozen sections can be performed in less than 15 minutes, preserving sufficient amounts of mRNA to allow detection of cell specific mRNAs from as little as 200 microdissected cells with 40 cycles of amplification. ${ }^{24}$ Using cytospins of rat pituitary cells and a sensitive nested PCR technique, Jin et al amplified pituitary hormone mRNA from single immunostained captured cells. ${ }^{27}$ However, when compared with conventional stains, immunostaining always results in a measurable decrease in RNA recovery, probably because of the longer exposure to aqueous media. ${ }^{24} 27$

Because LCM helps to collect exactly determinable numbers of purified cells under controlled conditions, its combination with methods such as real time quantitative RTPCR will allow a more precise determination of cell specific gene expression on a microscopic scale. ${ }^{14} 4650$

To understand better the complexity of genetic changes in pathologically altered tissues, the simultaneous detection of multiple different messages is preferable over the examination of single or few expressed genes. Techniques such as cDNA array hybridisation or serial analysis of gene expression can help to establish characteristic expression profiles of normal and neoplastic cells..$^{51-53}$ Changes in gene expression related to the different steps of tumorigenesis could be revealed with techniques such as subtractive hybridisation or differential display, by comparing related lesions such as epithelial dyplasia, carcinoma in situ, and invasive carcinoma microdissected from a single tissue block. ${ }^{545}$

The feasibility of combining LCM and cDNA microarray hybridisation has been demonstrated by Luo et al, who showed reproducible differences in gene expression between large and small neurones isolated from rat dorsal root ganglia. For each experiment, 1000 cells of one population were captured, and the RNA was amplified with T7 RNA polymerase to obtain sufficient material to generate the fluorescent probe for microarray hybridisation. ${ }^{26} \mathrm{~A}$ similar approach, combining LCM, cDNA arrays, and real time quantitative PCR, was used to show altered gene expression patterns at various stages of breast cancer progression. ${ }^{50}$

As mentioned above, LCM can also be used to obtain expression libraries from purified cell populations for CGAP. ${ }^{56}$ Several libraries derived from microdissected normal, preneoplastic, and neoplastic breast, prostate, ovary, lung, and liver tissues with large numbers of partially sequenced clones are already available at the CGAP web site (http:// www.ncbi.nlm.nih.gov/ncicgap). The power of LCM for creating tissue specific expression libraries has recently been demonstrated by studies aimed at the identification of prostate specific genes by the analysis of expressed sequence tags (ESTs). A highly expressed T cell receptor $\gamma$ transcript found in prostate libraries generated from microdissected tissue was initially believed to stem from contaminating $\mathrm{T}$ cells in the prostatic interstitium. However, subsequent studies showed that the transcript did originate from prostate epithelial cells. ${ }^{57}$ Similar surprising results running contrary to known tissue expression patterns can be expected in the future from this cell specific approach to genetic analysis. To produce useful information, the quality of primary tissues used for large scale expression studies is of utmost importance, and tissue procurement, time to freezing, precision of microdissection, and other parameters need to be controlled rigorously.

\section{SINGLE CELL ANALYSIS}

Although single cell analysis was introduced to pathology and other fields of biomedical research before the advent of laser based microdissection techniques and has allowed some spectacular progress, such as the identification of the lineage and clonality of Hodgkin's disease or the identification of genetic disorders in in vitro fertilised oocytes before implantation, the dedication and manual dexterity required for micromanipulation based microdissection precluded the wide use of this technique. ${ }^{59}{ }^{60}$ Both LCM and LMM have been applied successfully to the isolation of single cells. Point mutations in oncogenes such as RAS, viral DNA sequences, or rearranged immunoglobulin gene sequences have been identified from microdissected single cells, and the amplification of expressed gene sequences by RT-PCR has been performed successfully from both fresh and paraffin wax embedded tissues. ${ }^{11} 1214152729$ A modification of LCM specifically designed for single cell capturing was developed by Suarez-Quian et al, who replaced the large cap surface with a cylinder covered with EVA polymer membrane. ${ }^{21}$ This reduces the contact area with the tissue to a $40 \mu \mathrm{m}$ wide strip, allowing more precise dissection and greatly reducing the risk of the non-specific adherence of cells. By lifting and stepwise rotation of the cylinder through a motorised arm to provide a new strip of mem- 
brane, a large number of cells can be collected on the surface, similarly to conventional LCM.

In addition to specific targets, single cells obtained by laser microdissection can be used as a template for whole genome amplification, the generation of expression libraries, or probes for expression profiling with cDNA arrays. ${ }^{17}{ }^{26}$

Single cell analysis poses special problems for microdissection and for subsequent molecular studies. The isolation of single cells from cytological preparations is relatively straightforward with both LCM and LMM because the cells are already physically separated and usually complete. In contrast, single cell isolation from tissue sections is influenced both by the properties of sectioned tissues and the technical limits of microdissection. Because of suboptimal optical resolution on uncovered sections, the cell borders are indistinct in routine stains, although cell nuclei are usually identifiable. This is crucially important if the cytoplasmic compartment (for example, mRNA) is analysed. Attached fragments of other cells may alter expression profiles greatly. Immunohistochemical membrane stains can help to circumvent this problem, but the precise recovery of cytoplasmic compartments without contamination from adjacent cells may be impossible if the cellular outlines are irregular. Furthermore, sectioning generates a large number of fragmented cells that lack parts of the cytoplasm or even the nucleus. Consequently, the analysis of sectioned cells result in considerable loss of genetic information so that multiple experiments should be performed. In addition, the high number of cycles of amplification necessary for single cell analysis increases the risk of artifacts through preferential amplification of certain templates as well as contamination.

Apart from these technical problems, single cell results might not be representative of the cell population as a whole because of events specific for the analysed cell, such as the position in the cell cycle or other random events. Therefore, for many applications the pooling of microdissected single cells is advocated to increase the representativity of the sample and the detection sensitivity.

\section{Future developments}

Because LCM is a relatively new technique, refinements and modifications of the general principle of "aim and shoot" can be expected. More precise single cell techniques will reduce the possibility of contamination when small numbers of cells are examined. ${ }^{21}$ Combining the LCM microscope with a computer controlled, programmable stage will make automated LCM of preselected areas possible, thus reducing the time needed for extensive tissue sampling even further.

Novel approaches to fixation and tissue embedding with avoidance of crosslinking fixatives, optimisation of rapid immunostaining protocols, and improvements in DNA and RNA extraction techniques will increase the yield of high quality nucleic acids from small cell samples and make "molecular histology" a reality. The wealth of information generated by the high throughput genetic analysis of microdissected samples using cDNA microarrays and the large scale sequencing of ESTs will require rigorous validation by other techniques, and the extraction of diagostically and prognostically useful information will pose a challenge to pathologists and researchers in other fields.

A further dimension of tissue analysis on a microscopic scale is the examination of proteins. Preliminary studies already demonstrated the feasibility of "proteomics" with two dimensional gel electrophoresis, immunoblotting, and zymography on cell samples obtained by LCM. ${ }^{16}{ }^{61-65}$

We are grateful to $M$ Emmert-Buck for critical reading of the manuscript.

1 Bianchi AB, Navone NM, Conti CJ. Detection of loss of heterozygosity in formalin-fixed, paraffin-embedded tumor specimens by the polymerase chain reaction. Am $\mathcal{F}$ Pathol 1991;138:279-84.

2 Deng G, Lu Y, Zlotnikov G, et al. Loss of heterozygosity in normal tissue adjacent to breast carcinomas. Science 1996; normal tissue

3 Hiller T, Snell L, Watson P. Microdissection RT-PCR analysis of gene expression in pathologically defined frozen tissues. Biotechniques 1996;21:38-44.

4 Kanzler H, Küppers R, Hansmann ML, et al. Hodgkin and Reed-Sternberg cells in Hodgkin's disease represent the outgrowth of a dominant tumor clone derived from crippled) germinal center B cells. f Exp Med 1996;184: 1495-505.

5 Küppers R, Rajewsky K, Zhao M, et al. Hodgkin's disease: Hodgkin and Reed-Sternberg cells picked from histological sections show clonal immunoglobulin gene rearrangements and appear to be derived from B cells at various stages of development. Proc Natl Acad Sci U S A 1994;91:10962-6.

6 Radford DM, Fair K, Thompson AM, et al. Allelic loss of Radford DM, Fair K, Thompson AM, et al. Allelic loss of
chromosome 17 in ductal carcinoma in situ of the breast. Chromosome 17 in ductal carcin

7 Whetsell L, Maw G, Nadon N, et al. Polymerase chain reaction microanalysis of tumors from stained histological slides. Oncogene 1992;7:2355-61.

8 Going JJ, Lamb RF. Practical histological microdissection for PCR analysis. $\mathcal{F}$ Pathol 1996;179:121-4

9 Moskaluk CA, Kern SE. Microdissection and polymerase chain reaction amplification of genomic DNA from histological tissue sections. Am f Pathol 1997;150:1547-52.

10 Zhuang Z, Bertheau P, Emmert-Buck MR, et al. A microdissection technique for archival DNA analysis of specific cell populations in lesions $<1 \mathrm{~mm}$ in size. $A m \mathcal{F}$ Pathol 1995;146:620-5.

11 Becker I, Becker K-F, Röhrl MH, et al. Single-cell mutation analysis of tumors from stained histologic slides. Lab Invest 1997;75:801-7.

12 Bernsen MR, Dijkman HBPM, de Vries E, et al. Identification of multiple mRNA and DNA sequences from small tissue samples isolated by laser-assisted microdissection. Lab Invest 1998;78:1267-73.

13 Böhm M, Wieland I, Schütze K, et al. Microbeam MOMenT: non-contact laser microdissection of membrane-mounted native tissue. Am F Pathol 1997;151: 63-7.

14 Fink L, Seeger W, Ermert L, et al. Real-time quantitative RT-PCR after laser-assisted cell picking. Nat Med 1998;4: 1329-33.

15 Schütze K, Lahr G. Identification of expressed genes by laser-mediated manipulation of single cells. Nat Biotechnol 1998;16:737-42.

16 Emmert-Buck MR, Bonner RF, Smith PD, et al. Laser capture microdissection. Science 1996;274:998-1001.

17 Dietmaier W, Hartmann A, Wallinger S, et al. Multiple mutation analyses in single tumor cells with improved mutation analyses in single tumor cells with improved

18 Bonner RF, Emmert-Buck M, Cole K, et al. Laser capture Bonner RF, Emmert-Buck M, Cole K, et al. Laser capture 278:1481-3.

19 Dean-Clower E, Vortmeyer AO, Bonner RF, et al. Microdissection-based genetic discovery and analysis applied to cancer progression. Cancer f Sci Am 1997;3: 259-65

20 Simone NL, Bonner RF, Gillespie JW, et al. Laser-capture microdissection: opening the microscopic frontier to molecular analysis. Trends Genet 1998;14:272-6.

21 Suarez-Quian CA, Goldstein SR, Pohida T, et al. Laser capture microdissection of single cells from complex tissues. Biotechniques 1999;26:328-35.

22 Strausberg RL, Dahl CA, Klausner RD. New opportunities for uncovering the molecular basis of cancer. Nat Genet

23 Goldstein SR, McQueen PG, Bonner RF. Thermal modeling of laser capture microdissection. Applied Optics 1998;37:7378-91. 
24 Fend F, Emmert-Buck MR, Chuaqui R, et al. ImmunoLCM: laser capture microdissection of immunostained frozen

25 Fend F, Quintanilla-Martinez L, Kumar S, et al. Composite low grade B-cell lymphomas with two immunophenotypically distinct cell populations are true biclonal lymphomas. A molecular analysis using laser capture microdissection. Am $\mathcal{F}$ Pathol 1999;154:1857-66.

26 Luo L, Salunga C, Guo H, et al. Gene expression profiles of laser-captured adjacent neuronal subtypes. Nat Med 1999 5:117-22.

27 Jin L, Thompson CA, Qian X, et al. Analysis of anterior pituitary hormone mRNA expression in immunophenotypically characterized single cells after laser capture microdissection. Lab Invest 1999;79:511-12.

28 Maitra A, Wistuba II, Virmani AK, et al. Enrichment of epithelial cells for molecular studies. Nat Med 1999;5:459-63.

29 Quintanilla-Martinez L, Fend F, Rodriguez Moguel L, et al. Peripheral T-cell lymphoma with Reed-Sternberg-like cells Peripheral T-cell lymphoma with Reed-Sternberg-like cells of B-cell phenotype and genotype associated with Epstein

30 Isenberg G, Bielser W, Meier-Ruge W, et al. Cell surgery by laser micro-dissection: a preparative method. 7 Micros 1976;107:19-24

31 Meier-Ruge W, Bielser W, Remy E, et al. The laser in the Lowry technique for microdissection of freeze-dried tissue slices. Histochem $\mathcal{F} 1976 ; 8: 387-401$

32 Vocke CD, Pozzatti RO, Bostwick DG, et al. Analysis of 99 microdissected prostate carcinomas reveals a high frequency of allelic loss on chromosome 8p12-21. Cancer Res 1996;56:2411-16.

33 Zhuang Z, Merino MJ, Chuaqui R, et al. Identical allelic loss on chromosome $11 \mathrm{q} 13$ in microdissected in situ and invasive breast cancer. Cancer Res 1995;55:467-71.

34 Zhuang Z, Vortmeyer AO, Mark EJ, et al. Barrett's esophagus: metaplastic cells with loss of heterozygosity at the APC gene locus are clonal precursors to invasive the APC gene locus are clonal precursors

35 Zhang L, Cui X, Schmitt K, et al. Whole genome amplification from a single cell: implications for genetic analysis. tion from a single cell: implications for g
Proc Natl Acad Sci U S A 1992;89:5847-51.

36 Aubele M, Zitzelsberger H, Schenck U, et al. Distinct cytogenetic alterations in squamous intraepithelial lesions of the cervix revealed by laser-assisted microdissection and comparative genomic hybridization. Cancer 1998;84:3759.

37 Kuukasjarvi T, Tanner M, Pennanen S, et al. Genetic changes in intraductal breast cancer detected by comparative genomic hybridization. Am f Pathol 1997;150:146571 .

38 Weber RG, Scheer M, Born IA, et al. Recurrent chromosomal imbalances detected in biopsy material from oral premalignant and malignant lesions by combined tissue premalignant and malignant lesions by combined tissue parative genomic hybridization. Am f Pathol 1998;153: parative $295-303$.

39 Chandrasekharappa SC, Guru SC, Manickam P, et al. Positional cloning of the gene for multiple endocrine neoplasiatype 1 . Science 1997;276:404-7.

40 Brown MR, Chuaqui R, Vocke CD, et al. Allelic loss on chromosome arm 8p: analysis of epithelial ovarian tumors. Gynecol Oncol 1999;74:98-102.

41 Darling TN, Yee C, Bauer JW, et al. Revertant mosaicism partial correction of a germ-line mutation in COL17A1 by a frame-restoring mutation. $\mathcal{F}$ Clin Invest 1999;103:1371-7.

42 Tam AS, Foley JF, Devereux TR, et al. High frequency and heterogeneous distribution of $\mathrm{p} 53$ mutations in aflatoxin B1 induced mouse lung tumours. Cancer Res 1999;59: 3634-40.

43 Lieberman R, Crowell JA, Hawk ET, et al. Development of new cancer chemoprevention agents: role of pharmacokinetic/pharmacodynamic and intermediate end-

44 To MD, Done SJ, Redston M, et al. Analysis of mRNA from microdissected frozen tissue sections without RNA isolamicrodissected frozen tissue sections
tion. Am f Pathol 1998;153:47-51.
45 Shibutani M, Uneyama C, Miyazaki K, et al. Methacarn fixation: a novel tool for analysis of gene expressions in paraffin-emb.

46 Goldsworthy SM, Stockton PS, Trempus CS, et al. Effects of fixation on RNA extraction and amplification from laser capture microdissected tissue. Mol Carcinog 1999;25:8691

47 Glasow A, Haidan A, Gillespie J, et al. Differential expression of prolactin receptor (PRLR) in normal and tumorous adrenal tissues: separation of cellular endocrine compartments by laser capture microdissection (LCM). Endocr Res 1998;24:857-62.

48 Glasow A, Haidan A, Hilbers U, et al. Expression of $\mathrm{Ob}$ receptor in normal human adrenals: differential regulation of adrenocortical and adrenomedullary function by leptin. f Clin Endocrinol Metab 1998;83:4459-66.

49 Kohda Y, Murakami H, Moe OW, et al. Analysis of segmental renal gene expression profile analysis of human breast cancer progression. Kidney Int 2000;57:321-31.

50 Sgroi DC, Teng S, Robinson G, et al. In vivo gene expression profile analysis of human breast cancer progression. Cancer Res 1999;59:5656-61.

51 DeRisi J, Penland L, Brown PO, et al. Use of a cDNA microarray to analyse gene expression patterns in human cancer. Nat Genet 1996;14:457-60.

52 Velculescu VE, Zhang L, Vogelstein B, et al. Serial analysis of gene expression. Science 1995;270:484-7.

53 Cossman J, Annunziata CM, Barash S, et al. ReedSternberg cell genome expression supports a B-cell lineage. Blood 1999:94:411-16.

54 Luqmani YA, Lymboura M. Subtraction hybridization cloning of RNA amplified from different cell populations 1994;222:102-9.

55 Liang P, Pardee AB. Differential display of eukaryotic messenger RNA by means of the polymerase chain reaction. Science 1992;257:967-71.

56 Peterson LA, Brown MR, Carlisle AJ, et al. An improved method for construction of directionally cloned cDNA libraries from microdissected cells. Cancer Res 1998;58: 5326-8.

57 Vasmatzis G, Essand M, Brinkmann U, et al. Discovery of three genes specifically expressed in human prostate by expressed sequence tag database analysis. Proc Natl Acad Sci U S A 1998;95:300-4.

58 Essand M, Vasmatzis G, Brinkmann U, et al. High expression of a specific T-cell receptor $\gamma$ transcript in prostate. Proc Natl Acad Sci US A 1999;96:9287-92

59 Küppers R, Zhao M, Hansmann ML, et al. Tracing B cell development in human germinal centres by molecular analysis of single cells picked from histological sections. EMBO F 1993;12:4955-67.

60 d'Amore F, Stribley JA, Ohno T, et al. Molecular studies on single cells harvested by micromanipulation from archival tissue sections previously stained by immunohistochemistry or nonisotopic in situ hybridization. Lab Invest 1997;76: 219-24.

61 Banks RE, Dunn MJ, Forbes MA, et al. The potential use of laser capture microdissection to selectively obtain distinct populations of cells for proteomic analysis-preliminary findings. Electrophoresis 1999;20:689-700.

62 Emmert-Buck MR, Gillespie JW, Paweletz CP, et al. An approach to proteomic analysis of tumors. Mol Carcinog 2000;27:158-65.

63 Ornstein DK, Englert C, Gillespie JW, et al. Characterisation of intracellular prostate-specific antigen from laser capture microdissected benign and malignant prostatic epithelium. Clin Cancer Res 2000;6:353-6.

64 Simone NL, Remaley AT, Charboneau L, et al. Sensitive immunoassay of tissue cell proteins procured by laser capture microdissection. Am f Pathol 2000;156:445-52.

65 Natkanam Y, Rouse RV, Zhu S, et al. Immunoblot analysis of CD34 expression in histologically diverse neoplasms. Am ₹ Pathol 2000;156:21-7. 